\title{
Textes posthumes VI : Leçon sur Sartre et Husserl
}

\author{
PAR ROBERT BRISART \\ Université Saint-Louis à Bruxelles, Université de Luxembourg
}

Résumé Issu d'une audition faite à Liège en mai 2009, ce texte analyse le texte de Sartre, «Une idée fondamentale de la phénoménologie de Husserl: l'intentionnalité ». D'abord, Brisart y approuve l'interprétation de Sartre suivant laquelle l'intentionnalité de Husserl marque, à l'encontre d'un certain mythe de l'intériorité, une profession de foi réaliste «dans sa version externaliste la plus forte ». Ensuite, il dénonce chez l'un et l'autre un mythe plus profond, constitutif du réalisme philosophique, qu'il dénomme le mythe de l'objet.

Mots-clefs Sartre, Husserl, intentionnalité, réalisme.

Il y a sans doute plusieurs manière d'aborder ce petit texte de Sartre daté de janvier 1939 mais qui a été plus que vraisemblablement rédigé en 1934 et qui peut dès lors être considéré comme l'expression d'un premier contact de Sartre avec la phénoménologie et plus précisément avec la philosophie de Husserl ${ }^{1}$.

On peur aborder ce texte comme une introduction à la philosophie de

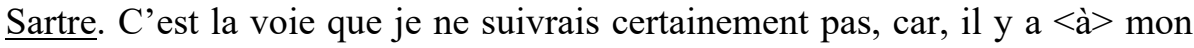
sens beaucoup mieux à en faire.

On peut également l'aborder dans le cadre d'une introduction au problème de l'intentionnalité et c'est la voie que je suivrais d'autant plus volontiers que j'ai appris par expérience tout l'intérêt « stratégique » à faire de

${ }^{1}$ L'auteur se réfère à J.-P. Sartre, « Une idée fondamentale de la phénoménologie de Husserl : l'intentionnalité », dans Situations I, Paris, Gallimard, 1947, p. 29-32. Voir la note de l'éditeur en fin de numéro. (N.d.l'É.) 
l'intentionnalité la topique fondamentale d'un cours de philosophie contemporaine, car elle ouvre directement sur ses productions essentielles tant continentales qu' anglo-saxonnes.

La question se pose dès lors de savoir ce que ce texte du tout jeune Sartre (il l'écrit à 28 ou 29 ans) a à nous dire à propos de l'intentionnalité et plus précisément à propos de l'usage husserlien de ce thème. Autrement dit : qu'y a-t-il à retenir de Sartre lecteur de Husserl, du moins sur la base de ce texte puisque la lecture sartrienne de Husserl semble avoir quelque peu, voire assez fortement, différé par la suite <alors même $>$ que certains diront que c'est dans le petit texte de 1934 (comme aussi bien sûr dans celui un peu plus étoffé $L a$ transcendance de l'ego qui lui est contemporain) que doivent être cherchées toutes les clés qui ouvrent le grand-œuvre de $1943:$ L'Être et le néant < ?>

A. Afin de répondre le plus clairement possible à cette question, je ne fonctionnerais pas comme l'ont bien souvent fait les commentateurs qui pratiquent une lecture à rebours, éclairant le texte de 34 à partir du livre de 43 en injectant donc pour ce faire certaines notions typiquement existentialistes que le texte qui nous occupe ne contient pas. Pour ma part, j'attirerais tout d'abord l'attention des étudiants sur deux thèses emblématiques qui se dégagent clairement du texte et je m'appliquerai bien sûr, dans un premier mouvement, à les développer :

1. la déconstruction du mythe de l'intériorité

2 la protestation de réalisme en philosophie

B. Dans un second mouvement, j'attirerais leur attention sur le fait que, nonobstant les critiques multiples qui ont été faites des quatre pages sur Husserl écrites par Sartre en 1934, celui-ci est allé pour ainsi dire à l'essentiel et, tout compte fait, a vu juste. N'aurait-il pratiqué qu'une lecture très superficielle de la phénoménologie comme on le lui a amplement reproché, Sartre ne s'est pas pour autant trompé sur la nature profonde de l'idée fondamentale de l'intentionnalité husserlienne :

— la conception husserlienne de l'intentionnalité entend bien renouer avec le réalisme

— du même coup, elle épouse une position qui ne saurait reconnaître au sujet aucune prérogative sur l'objet ou sur le monde réel

C. Dans un troisième mouvement, mais dont le développement devrait faire l'objet de plusieurs leçons à suivre, je poserais aux étudiants la question de savoir si le réalisme qui nous est présenté ici dans sa version externaliste la

\section{2}

Bull. anal. phén. XV 8 (2019)

https://popups.uliege.be/1782-2041/ @ 2019 ULiège BAP 
plus forte, constitue une position philosophique tout simplement tenable ? Car je nourris plus d'un doute à cet égard, je leur expliquerais les quelques bonnes raisons que nous avons de renvoyer Sartre et Husserl dos à dos : non pas certes pour nous passer de l'intentionnalité, mais, parce que nous ne pouvons nous en passer, pour repenser l'idée d'intentionnalité dans l'héritage de la révolution kantienne, alors que Sartre nous montre plutôt, et à juste titre, comment Husserl s'est employé à extirper la conscience de cet héritage par les moyens de l'intentionnalité.

Comme c'est très précisément sur ce point que débute notre texte de 1934, j'en viens donc au premier mouvement de mon exposé : la thèse de Sartre concernant l'intentionnalité.

Pour en préparer l'exposition, il n'est, me semble-t-il, que de s'en remettre au texte lui-même. À l'encontre du subjectivisme idéaliste ou de l'intellectualisme qui domine alors l'université française et qu'incarne en particulier la philosophie de Léon Brunschvicg, Sartre voit dans la phénoménologie les prémisses d'un style philosophique nouveau où les choses et le monde ne sont plus traités à travers les concepts, les images mentales ou plus simplement les représentations que nous en avons, mais sont désormais traités pour eux-mêmes. La philosophie héritée de Descartes, mais surtout de Kant, c'est celle pour laquelle connaître les choses voulait dire les déglutir, les réduire à la substance même de la pensée, les assimiler à des contenus de conscience. Cette philosophie arachnéenne ne s'est jamais employée à autre chose que d'attirer tout ce qui est dans la toile de la pensée et par ses propres sucs tout transformé en idées ou en concept, c'est-à-dire en un produit de la pensée elle-même. À l'inverse, ce que Sartre considère dans la phénoménologie, c'est cette philosophie qui veut rendre leurs droits aux choses et au monde ; c'est cette philosophie qui n'a pour seul mot d'ordre que le retour à la chose même, "Zu den Sachen selbst ».

Afin d'éclairer si besoin en est cette idée, pourquoi ne pas $<$ se $>$ servir d'images, les étudiants se sentent souvent mieux éclairés par elles. Par rapport au petit tableau de Rembrandt intitulé le philosophe en méditation, à suivre Sartre ce serait un tout autre tableau que nous suggère la pensée de Husserl. Dans la toile de Rembrandt, le philosophe se tient près de sa fenêtre, mais malgré la lumière assez forte, trop forte sans doute, qu'elle laisse passer $<,>$ ce n'est pas vers la fenêtre, ce n'est donc pas vers le monde qu'est tourné le regard du philosophe. Il est plutôt résolument tourné vers cet autre foyer de lumière sur la toile qu'est l'âtre, lumière plus douce, moins aveuglante, mais aussi plus familière, plus intime. Car cet âtre ne peut être que l'image de l'intériorité ou de la conscience elle-même, c'est bien, comme on l'a toujours dit, l'esprit de la philosophie moderne qu'a su rendre Rembrandt. Avec le Husserl de Sartre, 
tout se passe comme si, non seulement, le philosophe tournait son regard vers la fenêtre et affrontait la lumière aveuglante, mais par ce mouvement voyait aussi sa propre pensée projetée vers le monde, en quelque sorte contrainte à la défenestration.

Pour Sartre c'est précisément ce que réalise l'interprétation phénoménologique de la conscience en terme d'intentionnalité. Soutenir en effet que l'essence des actes $<$ de $>$ conscience est de nature intentionnelle, c'est soutenir que le pouvoir de la conscience ne se mesure pas à ses facultés de représentation, à sa force d'intégration des choses dans le moule immanent du psychique mais qu'inversement elle se mesure à sa puissance d'ouverture au monde extérieur, à sa transcendance. Pour Sartre, c'est ce que signifie clairement la thèse que toute conscience est conscience de quelque chose. En d'autres termes, qu'il soit perceptif, imaginant, désirant, judicatif ou affectif, tout acte de conscience vise un objet transcendant. Certes, il n'est pas toujours atteint car cet objet est parfois irréel, fictif, voire impossible ou absurde, mais l'important est que quelque soit l'objet, réel ou irréel, qu'il soit donc atteint ou non, c'est bien vers lui qu'est tendue la conscience en chacun de ses actes et non pas vers des contenus immanents, des images ou des représentations. Étant de nature intentionnelle, la conscience n'est pas vouée à n'avoir affaire qu'à elle-même, elle est au contraire sans cesse tournée vers le monde extérieur. Ainsi, pour Sartre, la découverte phénoménologique de l'intentionnalité constitue la promesse d'une redécouverte du monde dans toute la richesse de ses qualités redevenues objectives ; pour reprendre l'expression sartrienne : l'intentionnalité de la conscience réinstalle "l'horreur et le charme dans les choses » dans le même mouvement qu'elle permet de se défaire d'une conception de la conscience qui l' assimile à une boîte à l'intérieur de laquelle l'objet n'est plus qu'un contenu de représentation formé par le sujet lui-même. Contre cette philosophie « digestive », la leçon de l'intentionnalité est au contraire que « la conscience n'a pas de dedans ».

Nous touchons donc là à la première thèse qu'il m'importerait de faire ressortir auprès des étudiants : la liquidation du mythe de l'intériorité. Comprenons-la bien cependant. Par cette liquidation, il ne s'agit certainement pas pour Sartre de vouloir en revenir à l'attitude naturelle qui n'a d'yeux que pour ce qui nous entoure. En d'autres mots, il ne s'agit pas de renoncer à la réflexivité de la conscience, ce qui reviendrait peu ou prou à donner son congé à la philosophie. Mais ce que révèle justement la réflexion quand elle s'exerce, c'est qu'en chacun de ses actes la conscience est tendue vers les choses ; par chacun de ses actes elle est au monde. À la réflexion, l'intériorité ne livre que le dehors auquel est vouée la conscience. Comme le dit Sartre, revenir au- 
dedans c'est être « saisi par un tourbillon et aussitôt rejeté au-dehors ». Considérer le dedans ou l'homme intérieur comme autre chose que sa continuelle expulsion vers les choses, et prétendre y trouver quelques traces d'immanence, y trouver comme un reste d'irréductibilité par rapport au dehors, c'est tout simplement un mythe et pour Sartre justement un mythe que la phénoménologie nous autorise, plus, nous oblige à liquider.

Mais, comme je l'ai dit, cette première thèse en laisse courir une autre, moins perceptible dans le texte lui-même, donc, dirais-je plus subtile aussi. Cette seconde thèse tient dans la promotion sartrienne du réalisme. Certes n'importe quel étudiant qui a lu le texte un tant soit peu attentivement m'objectera que Sartre y affirme bien que « Husserl n'est point réaliste » et qu'il y a donc peu de chance que Sartre ne le suive pas sur ce point aussi. Mais sur quoi porte ici la dénégation de réalisme chez Sartre lecteur de Husserl ? Simplement sur le fait que dire de la chose qu'elle n'est pas ce qu'en fait la conscience n'équivaut pas à dire de la chose qu'elle est sans relation à la conscience. C'est ce que dément précisément l'intentionnalité : la chose est partout le corrélat de la conscience et il n'y a pas de conscience sans cette corrélation à un objet. Le déni de réalisme est donc ici le déni de l'absolutisme des positions. Il consiste à dire qu'il n'y a pas de face à face du sujet et de l'objet, mais une relativité de l'un par rapport à l'autre et c'est précisément cela que traduit l'intentionnalité. Mais dire qu'il y a rapport intentionnel à l'objet, ce n'est en rien préjuger de la nature de ce rapport, car il se pourrait fort bien que ce rapport soit d'ordre constitutionnel (le rapport consisterait alors à constituer l'objet) ou au contraire que ce rapport soit non constitutionnel parce que l'objet est supposé déjà constitué ou si l'on préfère donné tel quel. Or c'est la seconde alternative qui détermine ce que nous entendons par réalisme en philosophie. À l'étudiant imaginaire qui m'aurait soulevé l'objection, je répondrais donc assez volontiers que la dénégation sartrienne du réalisme n'atteint nullement ce que nous entendons par ce terme lorsque nous l'utilisons en philosophie pour qualifier cette position ontologique assez forte selon laquelle il y aurait un donné, un monde d'objets déjà constitués auquel notre conscience ne fait que se rapporter. Or, à mieux y regarder, ce réalisme ontologique, n'est-il pas celui qu'entraîne la déconstruction sartrienne du mythe de l'intériorité ? Je pense que oui. Si, comme je l'aurai montré à nos étudiants, la transcendance ou l'être au monde de la conscience est sans reste, si, comme le soutient Sartre, il n'existe absolument rien qui, par-devers elle, puisse passer comme principe ou comme loi de constitution de l'objectivité, sinon de récidiver alors dans le mythe de l'intériorité, alors c'est bien une forme assez forte de réalisme qu'implique l'interprétation sartrienne de la notion d'intentionnalité. Ce réalisme se mesure au poids ontologique du dehors 
qui détermine de part en part la transcendance de la conscience et lui dérobe du même coup toute possibilité de se réclamer du titre de conscience transcendantale, c'est-à-dire de conscience constituante de l'objet. C'est au contraire l'être du dehors qui fonde l'être du Cogito. En ce sens, peut-on dire, le réalisme sartrien de 1934 s'apparente à un externalisme radical, qui, d'exposer tout entière la conscience au dehors, la laisse entièrement dépendante d'un être ou d'une réalité qui échappe à sa maîtrise et auquel l'ouverture devient donc synonyme d'aventure sous « l'aveuglante lumière » comme le dit Sartre.

Peu de commentateurs ont pointé ce réalisme sartrien, si ce n'est peutêtre Lévinas qui précisément en a fait le lieu d'une véritable trahison par rapport à Husserl. Nous touchons là au second mouvement de la leçon : Sartre, lecteur de Husserl, lui est-il resté fidèle ? Je vous ai déjà fait part de ce que serait en l'occurrence ma réponse à la question : contrairement à Levinas, j'en suis venu à penser que oui, oui en tout cas pour l'essentiel. Ce qui peut surprendre et devrait en tout cas surprendre des étudiants de $3^{\mathrm{e}}$ BAC qui, ne disposant même que d'une brève initiation à la phénoménologie husserlienne, en savent en tout cas déjà assez pour m'objecter plusieurs choses. Ces étudiants, sans doute très hypothétiques, concéderont certes que c'est contre une philosophie immanentiste ou mentaliste, celle de Brentano que Husserl s'est réapproprié la notion d'intentionnalité pour marquer avec elle la relation des actes de conscience non pas vers des représentations mentales mais bien vers les objets extérieurs. Et pour sûr, il n'est qu'à lire l'appendice aux $\S \S 11$ et 20 de la cinquième Recherche logique pour se convaincre que la notion husserlienne d'intentionnalité a procédé elle aussi d'une dénonciation du mythe de l'intériorité entretenu par la théorie des images, ou la théorie des objets immanents, propre à ces philosophies qualifiées de son côté par Sartre de digestives. Mais cette concession étant faite, mes étudiants hypothétiques me diront qu'il n'est décidément pas possible d'aller plus loin. Pourquoi ? Parce que, répondront-ils, en réduisant l'intentionnalité à l'idée que toute conscience est toujours conscience de quelque chose - idée qui n'exprime rien de plus que la constante direction vers le monde qui fait tout l'être de la conscience , Sartre ne nous donne qu'une version scolastique de l'intentionnalité. Or, non seulement cette version fait l'impasse sur tout le dispositif descriptif des constituants intentionnels déployé par Husserl, mais du même coup replonge dans l'attitude naturelle contre laquelle veut nous prémunir la réduction phénoménologique dont d'ailleurs Sartre ne souffle mot dans son texte de 1934.

Puisqu'on m'a demandé de ne pas m'appesantir sur la pensée de Husserl, je me contenterai de dire ce que je répondrais à mes hypothétiques étudiants. Tout ce que vous dites est vrai, mais ne l'est que partiellement car 
vos objections émanent d'un certain niveau de l'analyse husserlienne, disons celui de la réduction phénoménologique. Ce faisant, vos objections laissent pour compte un niveau plus profond, celui du réalisme ontologique dont jamais, pas même dans les Ideen I, ne se départit la conception husserlienne de l'intentionnalité. Du coup, vos objections n'atteignent pas non plus l'interprétation elle-même profondément réaliste qu'en donne Sartre et qu'il peut nous donner du fait précisément de se passer de la réduction phénoménologique. Pour le moins, ma réponse mériterait une longue explication dont voici ce qui me paraît devoir être l'argumentaire essentiel exposé le plus brièvement possible en 4 points.

1. En la matière c'est de la notion de noème qu'il faut partir, puisque, de toute évidence, c'est ce concept devenu central dans la phénoménologie husserlienne à partir de 1908, qui a motivé la réaction de nos étudiants hypothétiques lorsqu'ils évoquaient la question des constituants de l'intentionnalité qui en rendrait la version sartrienne par trop simplificatrice, appauvrissante et au bout du compte parfaitement subversive.

2. Qu'est-ce donc que le noème ? Pour Husserl, il s'agit clairement de ce qui rend possible la directionnalité ou l'intentionnalité d'un acte de conscience vers un objet quel qu'il soit. Cette direction ne serait pas possible sans quelque chose comme une structure d'anticipation et de détermination qui nous permet d'identifier ce vers quoi ou le $\mathrm{X}$ vers lequel notre acte de conscience nous porte. Cette structure est le noème et elle équivaut au sens. En chaque cas, l'expérience de la conscience est donc reliée à un objet au moyen d'un noème ou d'un sens. Parce que ce sens est réitérable d'actes en actes, quelle que soit leur contingence spatio-temporelle, le sens ou le noème est une irréalité, une entité abstraite ou une idéalité en tout point comparable à ce que Frege appelait le Sinn.

3. La plus mauvaise interprétation que l'on puisse faire du noème, ou plus exactement du sens noématique, serait donc de le confondre avec l'objet de référence d'un acte intentionnel. C'est pourtant cette interprétation qui a toujours prévalu et Sartre lui-même y succombera lorsque, de sa première inspiration de 1934, il passera ultérieurement à une critique de l'immanentisme husserlien. Si celle-ci n'a pas lieu d'être, c'est que jamais Husserl ne rabat ni n'écrase la Bedeutung sur le Sinn, ou la référence sur le sens. C'est au demeurant la raison pour laquelle une lecture frégéenne de la phénoménologie demeure non seulement licite, mais de surcroît parfaitement éclairante. Le sens est bien le lieu où se constitue la relation à un objet, mais au demeurant, c'est l'objet et rien d'autre qui reste le référent ou le pôle directionnel des actes de conscience pour Husserl. 
4. Le noème n'est donc jamais ce que nous avons à l'esprit tant que nous vivons un acte de conscience ; cet acte est tout entier porté, dirigé vers son seul et véritable corrélat qu'est l'objet. Pourtant s'il y a bien une direction de l'acte vers l'objet, ce ne peut être que grâce au noème. Celui-ci doit donc être rendu explicite et, par là, en quelque sorte lui-même objectivé : ce qui ne se peut que si l'on quitte la spontanéité de l'acte pour adopter une attitude réflexive consistant en un acte tout à fait spécial que Husserl nomme la réduction phénoménologique. Avec elle, il s'agit donc seulement de mettre une sourdine à la relation effective des actes de conscience aux objets pour se porter uniquement du côté des actes eux-mêmes et, à l'intérieur de ceux-ci, mettre au jour la façon dont la direction vers l'objet est rendue possible moyennant le noème. La réduction phénoménologique ne sert donc qu'à montrer comment le noème constitue non pas l'objet, mais constitue la relation intentionnelle à l'objet. Comme le dit Husserl au $\S 15$ d'Ideen $I$ : « Dans le sens une relation à l'objet est constituée ».

De tout ceci, on conclura que jamais Husserl ne se départit de la thèse réaliste selon laquelle c'est bien aux objets transcendants que se rapporte la conscience, même si, dans ce rapport, celle-ci est parfois confrontée à des objets qui ne riment à rien. Cela fait au fond partie de l'aventure dont nous parlait Sartre qui, certes, simplifie à l'extrême pour ne pas faire le détour par la réduction phénoménologique, mais me semble pour autant ne rien retrancher à la position philosophique essentielle de Husserl. À cet égard, je dirais même que si le petit texte de 1934 feint d'ignorer, plutôt que de refuser, le grand détour par la problématique noématique, c'est pour mieux s'en tenir à l'essentiel de la thèse de Husserl concernant l'intentionnalité.

Pour que cette leçon ne reste pas sans lendemain, il resterait enfin à aborder le quatrième mouvement que je vous avais annoncé et amener les étudiants à réfléchir sur ce point : commentateur avisé de Husserl, Sartre estil en mesure pour autant d'apporter une solution avantageuse au problème fondamental de la phénoménologie, si par ce vocable on entend non pas un style, mais cette discipline de la philosophie qui a en charge d'explorer ce que veut dire expérience?

La question est évidemment pernicieuse, car, compte tenu de tout que j'aurai avancé, elle scelle le destin du commentaire de Sartre à l'œuvre de Husserl pour demander si l'un et l'autre ne cèdent pas à un mythe manifestement insoupçonné par eux-mêmes et qui me semble être le lot du réalisme philosophique en général, à savoir le mythe de l'objet. Selon ce mythe, quelque chose comme les objets ou comme le monde en général nous seraient donnés sans que nous ayons à les constituer ou à les fabriquer. J'aurai essayé de montrer aux étudiants que c'est là précisément l'idée à laquelle Sartre s'abandonne 
qui, dès le texte de 1934, se sert de la conception husserlienne de l'intentionnalité pour appuyer, d'une part, la massivité du réel et, d'autre part, la vacuité de la conscience. J'aurai ensuite attiré leur attention sur le fait que, si pour Husserl la conscience n'est pas si vide que cela, car il lui faut bien se ménager, par le sens, une voie d'accès au réel, celui-ci en tout cas s'impose comme ce que la conscience ne constitue pas, puisque c'est plutôt du réel que la conscience reste en attente d'un verdict de vérité concernant ses propres productions de sens, et par là de savoir si l'acte intentionnel a bien atteint son but. C'est donc en définitive la conception correspondantiste de la vérité telle qu'elle est corroborée par la fameuse théorie des remplissements qui pourrait le mieux servir à avérer la persistance du mythe de l'objet chez Husserl luimême.

À ce point, il resterait donc à demander si le délestage des prérogatives transcendantales que Sartre applique au sujet, n'est pas déjà le traitement que lui infligeait Husserl? De nouveau, la question a de quoi surprendre, car elle s'oppose à l'essentiel de la tradition interprétative de Husserl, mais elle me semble néanmoins fondée en regard de la seule interprétation de Sartre en 1934, si du moins cette interprétation est correcte, ce que je pense être le cas comme j'aurai tenté de le montrer.

Sur cette base, j'argumenterais enfin en faveur de l'idée qu'il ne saurait être question d'abandonner cette prérogative transcendantale si précisément il s'agit de prémunir la notion d'expérience contre le mythe réaliste de l'objet. $\mathrm{Si}$, du reste, quelqu'un comme John Searle nous montre dans le détail toutes bonnes raisons que nous avons de maintenir l'idée d'intentionnalité, je ne pourrais que terminer en indiquant aux étudiants l'intérêt que nous avons à réimplanter cette notion dans ce courant majeur de la philosophie moderne qui commence lorsque Kant échange la structure du monde pour la structure de l'esprit, qui continue avec Russell et C.I. Lewis lorsqu'ils échangent la structure de l'esprit pour la structure des concepts, et qui s'est poursuivie plus récemment avec Quine et Goodman par l'échange de la structure des concepts pour la structure des différents schèmes conceptuels qui déterminent l'entièreté de notre rapport aux objets que ce soit dans leur expérience perceptive quotidienne, que ce soit dans leur expérience épistémique proprement scientifique ou que ce soit encore dans leur expérience artistique. 


\section{Note de l'éditeur}

Ce texte de Robert Brisart - dont le titre complet est « Projet de leçon sur "Sartre, lecteur de Husserl" à partir de "Une idée fondamentale de la phénoménologie de Husserl : l'intentionnalité" " - trouve son origine dans une audition passée en 2009 alors qu'il briguait à Liège la chaire d'histoire de la philosophie contemporaine que devait laisser vacante, en septembre de la même année, le départ à la retraite de Daniel Giovannangeli. Réunie le mercredi $1^{\mathrm{er}}$ avril 2009, la commission d'attribution avait retenu quatre candidatures en vue de l'audition, qui eut lieu dans l'après-midi du 6 mai. La consigne était « d'exposer en une vingtaine de minutes les grandes lignes d'une leçon qu'il pourrait présenter à des étudiants de $3^{\mathrm{e}}$ bac sur le thème "Sartre, lecteur de Husserl : À partir du texte Une idée fondamentale de la phénoménologie de Husserl : l'intentionnalité (Situations I)" ». Brisart n'a pas décroché le poste. Ce projet de leçon est bien plus qu'un texte de circonstance, comme en témoigne le fait que son auteur l'a diffusé dans les jours qui ont suivi parmi plusieurs philosophes liégeois et qu'il a été alors abondamment discuté. Mes additions significatives sont signalées par des chevrons simples $(<>)$. (Denis Seron) 